\title{
Endoscopic drainage combined with percutaneous drainage in treatment of walled-off pancreatic necrosis - a single-center experience
}

\author{
Mateusz Jagielski ${ }^{1}$, Marian Smoczyński ${ }^{1}$, Michał Studniarek ${ }^{2}$, Krystian Adrych ${ }^{1}$ \\ ${ }^{1}$ Department of Gastroenterology and Hepatology, Medical University of Gdansk, Gdansk, Poland \\ 2Department of Radiology, Medical University of Gdansk, Gdansk, Poland \\ Gastroenterology Rev 2018; 13 (2): 137-142 \\ DOI: https://doi.org/10.5114/pg.2018.72604
}

Key words: endoscopic drainage, percutaneous drainage, pancreatic necrosis.

Address for correspondence: Mateusz Jagielski PhD, Department of Gastroenterology and Hepatology, Medical University of Gdansk, 17 Smoluchowskiego St, 80-214 Gdansk, Poland, phone: +48 5834936 40, fax: +48 58349 36 50, e-mail: matjagiel@gmail.com

\begin{abstract}
Introduction: In last three decades we have been observing development of minimally invasive walled-off pancreatic necrosis (WOPN) treatment techniques. The choice of access to the necrosis and technique of treatment depends not only on the position and spread of necrosis, but in the first place on the experience of the medical center.

Aim: To assess the effectiveness and safety of combined endoscopic and percutaneous drainage of WOPN.

Material and methods: We performed a retrospective analysis of 64 consecutive patients with symptomatic WOPN, who underwent endoscopic treatment in our department between 2011 and 2013.

Results: Additional percutaneous drainage was executed during endoscopic treatment in 20/64 (31.25\%) patients. Complications of treatment occurred in 4/20 (20\%) patients. Complications of treatment occurred in 4/20 (20\%) patients. All these complications were related to endoscopic treatment. No complications related to percutaneous drainage were noted. There were no deaths. Therapeutic success was achieved in all 20 patients. No patients required surgery. The average time of endoscopic drainage was 41.4 (11-173) days. The mean number of endoscopic procedures was $4.2(2-12)$. The average time of percutaneous drainage was 11.3 (5-20) days. The medium time of follow-up was 54 (48-64) months. During the observation the recurrence of WOPN was noted in 2/20 (10\%) patients. Long-term success of combined drainage was achieved in 18/20 (90\%) patients.

Conclusions: In selected patients with symptomatic WOPN combined endoscopic and percutaneous drainage enables a high success rate with a low procedure-related complication rate.
\end{abstract}

\section{Introduction}

In the course of acute necrotizing pancreatitis (ANP) it often leads to creation of pancreatic fluid collections (PFCs) [1, 2]. Necrosis of pancreatic parenchyma (as well as necrosis of peripancreatic tissues) in the first 4 weeks of ANP used to be described as acute necrotic collection (ANC), while after four weeks of disease it is named walled-off pancreatic necrosis (WOPN) [1, 2]. Walled-off pancreatic necrosis occurs in $49-58 \%$ of patients with ANP $[3,4]$.

The clinical suspicion or documented infection of pancreatic necrosis and clinical symptoms related to the presence of necrotic collection are the indications for the beginning of interventional WOPN treatment [5-7]. Utilization of minimally invasive techniques im- proves the results of treatment of patients with WOPN [8]. Minimally invasive methods include procedures performed with an endoscope, laparoscope, and nephroscope that enable transperitoneal, retroperitoneal, transmural or transpapillary access to necrosis $[5,9,10]$. Percutaneous drainage (PCD) allows transperitoneal or retroperitoneal access to the collection of WOPN $[5,10]$. Performance of PCD consists in insertion of a drain into the area of necrosis under ultrasonographic or computed tomography guidance $[5,10]$. Thereafter the drain is flushed with saline solution $[5,10]$. Percutaneous drainage was described for the first time in 1998 by Freeny et al., who used this drainage technique in 34 patients [11]. A beneficial effect of treatment was obtained in $47 \%$ of patients with a complication rate of $71 \%$ and mortality of $12 \%$ [11]. Endoscopic treatment of WOPN 


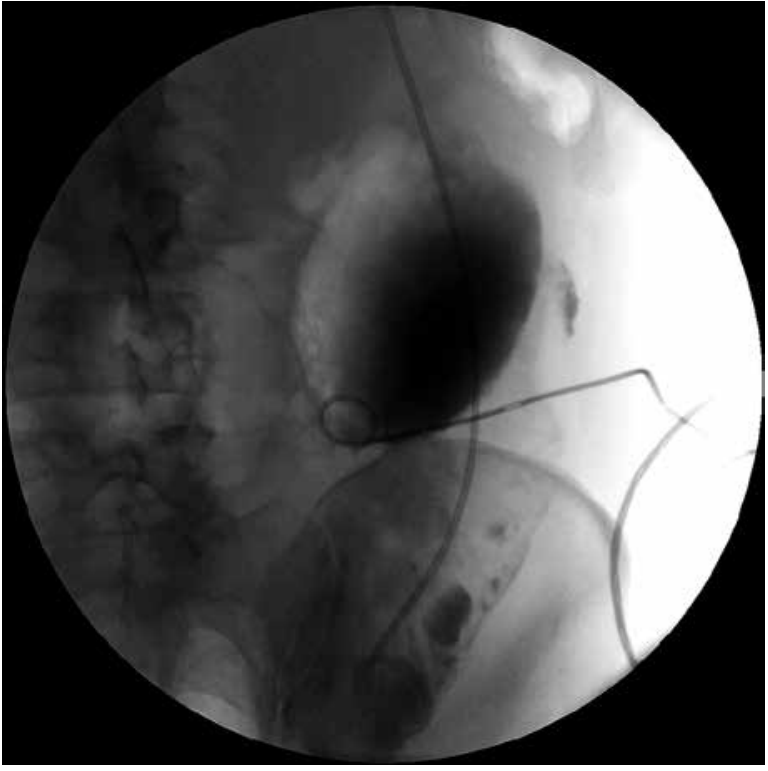

Figure 1. Fluoroscopic image shows endoscopic transmural drainage combined with percutaneous drainage in a patient with pancreatic necrosis

consists in transmural drainage (through the gastric or duodenal wall), transpapillary drainage or a combination of both methods of access to the necrotic cavity $[12,13]$. In 1996 Baron et al. became the first in the world to describe the results of efficient endoscopic treatment (transmural and transpapillary) of 11 patients suffering from pancreatic necrosis [12]. In 2010 Ross et al. presented the outcomes from the treatment of 15 patients with WOPN, in whom combined endoscopic and percutaneous drainage was used [14]. The same group of authors named this method in later publications dual-modality drainage $[15,16]$.

\section{Aim}

The aim of this study was to assess the effectiveness and safety of combined endoscopic and percutaneous drainage of WOPN. Herein we introduce the results of treatment of 20 patients with symptomatic WOPN, who were treated between 2011 and 2013 in our medical center with use of percutaneous drainage.

\section{Material and methods}

Sixty-four consecutive patients with symptomatic WOPN underwent endoscopic treatment in our department between 2011 and 2013.

Endoscopic drainage of WOPN has been applied in our medical center since 2001 [17, 18]. Conventional drainage (without endoscopic ultrasonography - EUS) was performed between 2001 and 2011 [17, 18]. Then the fistula was created on the top of the largest bulge into the gastric or duodenal wall. When the bulge was not visible enough, the location of the fistula was chosen on the basis of computed tomography and fluoroscopic images of the main pancreatic duct leak or the flow of contrast medium administered through the percutaneous drain. Percutaneous drainage during this time was most often (15 of 19 patients with PCD) exploited to administer the contrast through the drain and to show the necrotic collections in the fluoroscopic image in order to choose the best location of the fistula - particularly when the bulging of the necrotic collection was not visible endoscopically.

Since 2011 a fistula between the lumen of the gastrointestinal tract and the lumen of the necrotic collection has been performed under control of EUS (EUS-guided drainage) [18]. In our medical center percutaneous drainage was used for treatment of WOPN as an extra access to the necrotic collection during endoscopic treatment most intensively between 2011 and 2013. The development of endoscopic techniques of WOPN treatment has been significantly reducing the use of PCD since 2013. This paper presents a retrospective analysis of 20 patients with symptomatic WOPN, in whom percutaneous drainage was performed during the endoscopic treatment (Figure 1) between the years 2011-2013.

The qualification for minimally invasive treatment of pancreatic necrosis was based on the clinical picture and the results of contrast-enhanced computed tomography (CECT). The WOPN was diagnosed on the basis of the revised Atlanta classification issued in 2012 [1, 2]. The presence of necrotic debris in the EUS image and morphology of aspirated fluid taken from the collection (dark brown color and fragments of necrotic tissues) were used to confirm the diagnosis of WOPN.

Interventional treatment of WOPN was begun in each patient from the endoscopic drainage. Prior to it each patient received antibiotics (ciprofloxacin or ceftriaxone with metronidazole). Prophylactic antibiotic therapy was continued for 2 weeks. In the case of clinical symptoms indicating infection of the collection, antibiotic therapy was prolonged or modified on the basis of repeated microbial culture of fluid from the collection.

Endoscopic procedures were conducted using a Pentax ED3490TK duodenoscope and a Pentax EG3870UTK echoendoscope. In all patients an attempt of transmural drainage was made. The place of the transmural tract was chosen with the support of EUS guidance. Gastrostomy (or duodenostomy) was formed using a Giovannini cystostome (Wilson-Cook). The stoma made between the lumen of the gastrointestinal tract and necrotic cavity was dilated with an 8-mm or 20-mm balloon dilator (Boston Scientific). A 7 Fr or 8 Fr nasocystic drain (Bal- 
ton or Wilson-Cook) and double-pigtail stents $7 \mathrm{Fr}$ or 8.5 Fr (Wilson Cook or Mar Flow) were deployed within the necrotic cavity through the transmural tract.

After the endoscopic procedure the nasal drains were flushed with saline solution $(60-200 \mathrm{ml})$ every $2 \mathrm{~h}$ during the first $48 \mathrm{~h}$ and thereafter every $4 \mathrm{~h}$. The effect of endoscopic drainage was monitored every week, mainly using conventional ultrasonography. In the case of ineffective endoscopic drainage and spreading of necrosis outside the lesser omental sac, additional percutaneous drainage was performed.

Percutaneous drainage was made under ultrasonographic or computed tomography guidance. Retroperitoneal access to the necrosis was preferred. The transperitoneal approach was used in selected cases, when there was no technical opportunity to use the retroperitoneal approach. The site and method of PCD were chosen on the basis of the position and size of the WOPN. The tandem trocar technique was used for placement of the drains (6-10 Fr Wilson Cook or Boston Scientific). The access to the necrotic cavity was made by means of ultrasonographic or computed tomography guidance. The free-hand technique was used for placement of the drains into the lumen of the WOPN. One drain was used for each access site and the catheters were left for gravidity drainage and irrigated with saline solution (50-100 ml 3-4 times a day). The percutaneous drain was removed before the end of endoscopic drainage. After clamping the percutaneous drain for $24 \mathrm{~h}$ and verification of the absence of relapse of fluid collection, contrast-enhanced CT was done to confirm complete regression of the necrotic collection. Active endoscopic drainage was finished after resolution of clinical symptoms and complete regression of the collection or decrease of the collection's diameter to less than $30 \mathrm{~mm}$.

Therapeutic success was defined as a lack of symptoms and complete regression of the collection or the dimension of the collection less than $30 \mathrm{~mm}$ during a three-month follow-up from the end of active drainage. Recurrence of the collection was determined as the collection size $>30 \mathrm{~mm}$ or relapse of symptoms during a follow-up. Long-term success was defined as a lack of symptoms and complete regression of the collection or the dimension of the collection less than $30 \mathrm{~mm}$ during a follow-up.

\section{Statistical analysis}

All the statistical calculations were performed using the data analysis software system StatSoft Inc. (2011) Statistica version 10.0 (licensed for the Medical University of Gdansk). Quantitative variables were characterized by arithmetic means and minimal and maximal val- ues (range), whereas qualitative data were presented as means of numbers and percentage.

\section{Results}

Sixty-four consecutive patients with symptomatic WOPN underwent endoscopic treatment in our department between 2011 and 2013. Additional percutaneous drainage was executed during endoscopic treatment in 20/64 (31.25\%) patients (Figures 2 A-D). Demographic and clinical characteristics of this group of patients are presented in Table I.

The indications for the beginning of endoscopic WOPN treatment in the study group were as follows: gastrointestinal obstruction together with weight loss (13/20 patients), jaundice (3/20 patients), and clinical suspicion of infected WOPN (8/20 patients). At least 2 indications for the beginning of treatment were stated in 4 patients.

Walled-off pancreatic necrosis infection was diagnosed on the basis of positive microbial culture in all $8 / 20$ (40\%) patients in whom infection was suspected. The most common pathogens cultured in the necrotic contents were Escherichia coli, Enterococcus faecalis and Staphylococcus epidermidis. In 2/20 (10\%) patients sepsis with positive blood culture was observed during drainage.

Endoscopic transmural access was used in 19/20 (95\%) patients (gastric - 14, duodenal - 5). In 1 patient the transmural route was not used, because the distance between the gastrointestinal lumen and the necrotic cavity exceeded $15 \mathrm{~mm}$. Transpapillary access was used in this patient.

All 20 patients underwent an extra percutaneous drainage. The average time from the start of endoscopic treatment to the performance of percutaneous drainage was 13.9 days (4-39). One percutaneous catheter was inserted in 19 patients and 2 drains were inserted in 1 patient (Figure 3). Percutaneous drainage from the retroperitoneal approach was performed in 14 patients, while the PCD was done from the transperitoneal access in 6 patients (including 1 patient with 2 drains). Mean size of the catheter was $7.1 \mathrm{Fr}(6-10)$.

Complications of treatment occurred in 4/20 (20\%) patients. All these complications were related to endoscopic treatment (2 patients - upper gastrointestinal bleeding treated conservatively with packed red blood cell transfusions, 1 patient - gastrointestinal perforation treated conservatively and 1 patient - migration of transmural stent into the lumen of the WOPN treated endoscopically). No complications related to percutaneous drainage were noted. Furthermore, there were no complications in the form of a pancreaticocutaneous fistula. Also there were no deaths. 

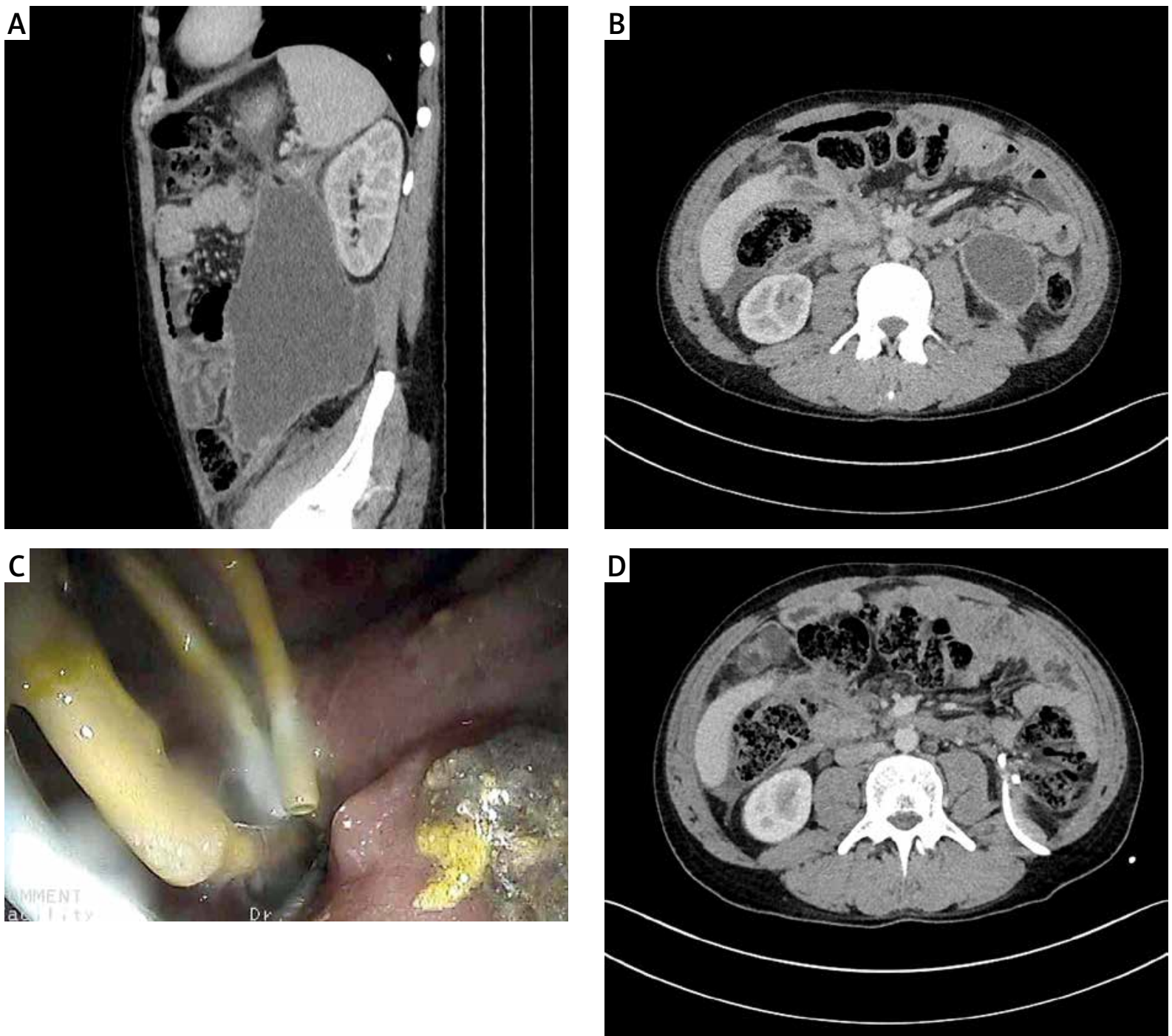

Figure 2. Contrast-enhanced computed tomography done before interventional treatment (A, B). Extensive WOPN collection is clearly visible. Endoscopic drainage (C) combined with percutaneous drainage was performed in this patient. Complete regression of necrosis was confirmed by CECT after 21 days of percutaneous drainage (D). Percutaneous catheter inserted retroperitoneally is visible on CT scan (D)

Therapeutic success was achieved in all 20 patients. No patients required surgery. The average time of endoscopic drainage was 41.4 days (11-173). The mean number of endoscopic procedures was 4.2 (2-12). The average time of percutaneous drainage was 11.3 days (5-20).

The medium time of follow-up was 54 (48-64) months. During the observation the recurrence of WOPN was noted in 2/20 (10\%) patients. Long-term success of combined drainage was achieved in 18/20 (90\%) patients.

\section{Discussion}

This retrospective study describes combined endoscopic and percutaneous drainage as a minimally inva- sive approach to symptomatic WOPN. We obtained in this highly selected group of patients very good results of treatment with a low rate of complications and number of recurrent fluid collections. What is more, there were no lethal complications, no pancreaticocutaneous fistulas and, most importantly, no surgery.

In 2010 Ross et al. became the first in the world to publish the results of treatment of 15 patients suffering from symptomatic WOPN, in whom combined endoscopic and percutaneous drainage was executed [14]. Ross et al. obtained favorable clinical outcomes with low procedure-related morbidity [14]. No lethal complications were noted and no patient required surgery [14]. The same authors described combined endoscopic 
Table I. Characteristics of the patients with WOPN $(n=20)$

\begin{tabular}{lc}
\hline Parameter & Value \\
\hline Age, mean (range) & $52.90(33-79)$ \\
\hline Sex, men, $n(\%)$ & $13(65)$ \\
\hline Etiology, $n(\%):$ & $12(60)$ \\
\hline Alcoholic & $8(40)$ \\
\hline Non-alcoholic & $14.6(6.5-25.3)$ \\
\hline $\begin{array}{l}\text { WOPN size, mean (range) [cm] } \\
\text { WOPN type, } n \text { (\%) }\end{array}$ & $2(10)$ \\
\hline $\begin{array}{l}\text { Pancreatic parenchymal necrosis alone } \\
\text { Both pancreatic and peripancreatic } \\
\text { necrosis }\end{array}$ & $20.30(4-60)$ \\
\hline $\begin{array}{l}\text { Time from the acute bout of pancreatitis, } \\
\text { mean (range) [weeks] }\end{array}$ &
\end{tabular}

and percutaneous drainage as dual-modality drainage in their subsequent publication [15, 16]. In 2011 Gluck et al. proved that dual-modality drainage decreases the amount of radiological and endoscopic procedures in patients with pancreatic necrosis comparing to standard percutaneous drainage. It also reduces the time of hospitalization and time of treatment [15].

In 2014 Ross et al. presented the outcomes of treatment of 117 patients with symptomatic WOPN, in whom dual-modality drainage was performed [16]. This method was successful in 103/117 (88.03\%) patients with complications in $6 / 117$ (5.13\%) of them and morbidity of $4 / 117$ (3.4\%) patients [16]. Four patients died from adverse events related to severe acute pancreatitis [16]. During the follow-up lasting 750 days recurrent fluid collections were noted in 8/103 (7.77\%) patients [16]. In our study the efficacy of treatment was $90 \%$ $(18 / 20)$ with a complication rate of $20 \%(4 / 20)$. No lethal complications were recorded and there was no need of surgical treatment. However, it is worth emphasizing that the better results in our publication are certainly related to the significantly lower number of patients who were treated this way.

The technique introduced in our paper differs (combined endoscopic and percutaneous drainage) from the method described as dual-modality drainage (DMD) [14-16]. First of all, the standard DMD consists in performance of percutaneous drainage, and endoscopic transmural drainage is done after it [14-16]. Herein endoscopic drainage is the basic treatment, and percutaneous drainage is done the next only in the case of lack of complete regression of WOPN. Furthermore, passive endoscopic drainage was used in the quoted studies [14-16]. It consisted in insertion of transmural stents

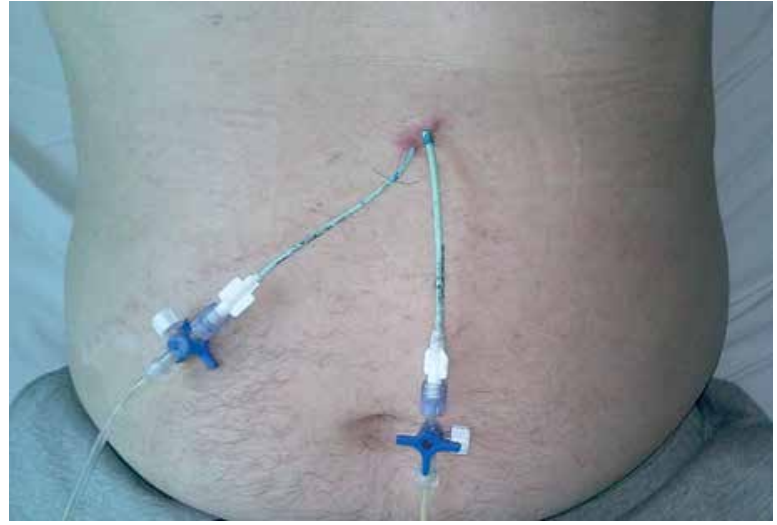

Figure 3. Image shows the patients with two percutaneous catheters guided transperitoneally into the area of WOPN

through the stoma, without a nasal drain [14-16]. Active endoscopic drainage which involves insertion of transmural endoprostheses as well as a nasal drain in order to flush the necrotic collection is done in every patient with WOPN in our medical center. In this way our technique is more aggressive, since we flush both nasal and percutaneous drains with saline solution.

Van Baal et al. described complications in $21.2 \%$ of patients, including pancreaticocutaneous fistulas (53\% of complications) and pancreaticoenteric fistulas (51.5\% of complications) [19]. None of the previously mentioned complications (pancreatic fistulas) were recorded in any studies concerning dual-modality drainage $[14,16]$, including our paper. In our opinion, the lack of such complications results from the small diameter of the percutaneous catheter (average 7.1 Fr) and short time of percutaneous drainage (average 11.3 days). The lack of such complications in the study of Ross et al. was explained by the authors by the role of transmural endoscopic drainage [16]. Transmural endoprostheses enable free outflow of pancreatic juice to the lumen of the gastrointestinal tract, reducing formation of pancreatic fistulas, particularly in patients with disconnected duct syndrome [16]. Transmural endoscopic drainage in our study might have been a key to success in prevention of creation of pancreatic fistulas in patients with WOPN, who were treated with combined endoscopic and percutaneous drainage.

This study has some limitations. The major ones are lack of randomization and the retrospective nature. Further limitations derive from the fact that it was conducted on the basis of a highly selected group of patients from a single medical center. The group of patients is also relatively small.

Nevertheless, endoscopic drainage combined with percutaneous drainage is an efficient method of treat- 
ment of patients with WOPN with an acceptable rate of complications. This technique is an alternative to other minimally invasive methods of treatment. We hold the view that the technique introduced here is useful when endoscopic treatment fails and still before definitive surgical treatment. In order to assess the utility of this technique more accurately, further multicenter and prospective studies conducted on a larger number of patients are needed.

\section{Conclusions}

In selected patients with symptomatic WOPN, combined endoscopic and percutaneous drainage enables a high success rate with a low procedure-related complication rate.

\section{Conflict of interest}

The authors declare no conflict of interest.

\section{References}

1. Banks PA, Bollen TL, Dervenis C, et al. Classification of acute pancreatitis - 2012: revision of the Atlanta classification and definitions by international consensus. Gut 2013; 62: 102-11.

2. Thoeni RF. The revised Atlanta classification of acute pancreatitis: its importance for the radiologist and its effect on treatment. Radiology 2012; 262: 751-64.

3. Sarathi Patra P, Das K, Bhattacharyya A, et al. Natural resolution or intervention for fluid collections in acute severe pancreatitis. Br J Surg 2014; 101: 1721-8.

4. Manrai M, Kochhar R, Gupta V, et al. Outcome of acute pancreatic and peripancreatic collections occurring in patients with acute pancreatitis. Ann Surg 2016 Epub ahead of print.

5. Freeman ML, Werner J, van Santvoort HC, et al. Interventions for necrotizing pancreatitis: summary of a multidisciplinary consensus conference. Pancreas 2012; 41: 1176-94.

6. Besselink M, van Santvoort H, Freeman M, et al.; Working Group IAP/APA Acute Pancreatitis Guidelines. IAP/APA evidence-based guidelines for the management of acute pancreatitis. Pancreatology 2013; 13: e1-15.

7. Gurusamy KS, Belgaumkar AP, Haswell A, et al. Interventions for necrotising pancreatitis. Cochrane Database Syst Rev 2016; 4: CD011383.

8. van Santvoort HC, Besselink MG, Bakker OJ, et al. A step-up approach or open necrosectomy for necrotizing pancreatitis. New Engl J of Med 2010; 362: 1491-502.

9. Loveday BP, Petrov MS, Connor S, et al. A comprehensive classification of invasive procedures for treating the local complications of acute pancreatitis based on visualization, route, and purpose. Pancreatology 2011; 11: 406-13.

10. Bello B, Matthews JB. Minimally invasive treatment of pancreatic necrosis. World J Gastroenterol 2012; 18: 6829-35.

11. Freeny PC, Hauptmann E, Althaus SJ, et al. Percutaneous CT-guided catheter drainage of infected acute necrotizing pancreatitis: techniques and results. AJR Am J Roentgenol 1998; 170: 969-75.
12. Baron TH, Thaggard WG, Morgan DE, Stanley RJ. Endoscopic therapy for organized pancreatic necrosis. Gastroenterology 1996; 111: 755-64.

13. Papachristou GI, Takahashi N, Chahal, et al. Peroral endoscopic drainage/debridement of walled-off pancreatic necrosis. Ann Surg 2007; 245: 943-51.

14. Ross A, Gluck M, Irani S, et al. Combined endoscopic and percutaneous drainage of organized pancreatic necrosis. Gastrointest Endosc 2010; 71: 79-84.

15. Gluck M, Ross A, Irani S, et al. Dual modality drainage for symptomatic walled-off pancreatic necrosis reduces length of hospitalization, radiological procedures, and number of endoscopies compared to standard percutaneous drainage. J Gastrointest Surg 2012; 16: 248-57.

16. Ross AS, Irani S, Gan SI, et al. Dual-modality drainage of infected and symptomatic walled-off pancreatic necrosis: long-term clinical outcomes. Gastrointest Endosc 2014; 79: 929-35.

17. Smoczyński M, Marek I, Dubowik M, et al. Endoscopic drainage/debridement of walled-off pancreatic necrosis - single center experience of 112 cases. Pancreatology 2014; 14: 137-42.

18. Jagielski M, Smoczyński M, Jabłońska A, et al. The role of endoscopic ultrasonography in endoscopic debridement of walled-off pancreatic necrosis - a single center experience. Pancreatology 2015; 15: 503-7.

19. van Baal MC, van Santvoort HC, Bollen TL, et al. Systematic review of percutaneous catheter drainage as primary treatment for necrotizing pancreatitis. Br J Surg 2011; 98: 18-27.

Received: 5.12.2017

Accepted: 11.12.2017 